\title{
Infra-Low Frequency Neurofeedback in Depression: Three Case Studies
}

\author{
Vera A. Grin-Yatsenko ${ }^{1 *}$, Siegfried Othmer ${ }^{2}$, Valery A. Ponomarev' ${ }^{1}$, Sergey A. Evdokimov, \\ Yuri Y. Konoplev ${ }^{3}$, and Juri D. Kropotov ${ }^{1}$ \\ ${ }^{1}$ N.P. Bechtereva Institute of the Human Brain of Russian Academy of Sciences, St. Petersburg, Russia \\ ${ }^{2} \mathrm{EEG}$ Institute, Woodland Hills, California, USA \\ ${ }^{3}$ Saint-Petersburg State University, Biology Faculty, Department of Higher Nervous Activity and Psychophysiology, St. \\ Petersburg, Russia
}

\begin{abstract}
Electroencephalographic (EEG) findings on depressive patients indicate theta and alpha activity higher than in normal controls. Extensive literature reports on the effectiveness of neurofeedback techniques in the treatment of cognitive and behavioral disorders by training the patients to modulate their brain activities, as reflected in their electroencephalogram. Three unmedicated, depressed individuals participated in this study of infra-low frequency neurofeedback (ILF NF) training. Along with the pre- and posttreatment Depression Rating Scales assessment, quantitative EEGs (qEEG) were recorded in eyes-open and eyes-closed resting states and during the visual cued Go/NoGo task before and after 20 sessions of training. Along with remission of the clinical symptoms of depression, significant decrease of theta power over frontal and central areas was observed in all three patients under all test conditions. These qEEG dynamics might be a correlate of ILF NF-related recovery of the appropriate level of frontal cortical activation.
\end{abstract}

Keywords: neurophysiology; neurofeedback; depression; qEEG; infra-low frequency

Citation: Grin-Yatsenko, V. A., Othmer, S., Ponomarev, V. A., Evdokimov, S. A., Konoplev, Y. Y., \& Kropotov, J. D. (2018). Infra-low frequency neurofeedback in depression: Three case studies. NeuroRegulation, 5(1), 30-42. http://dx.doi.org/10.15540/nr.5.1.30

*Address correspondence to: Vera A. Grin-Yatsenko, PhD, MD Laboratory of Neurobiology of Action Programming, Institute of the Human Brain of Russian Academy of Sciences, 197376, St. Petersburg, ul. Acad. Pavlova, 9, Russian Federation. Email: veragrin@yahoo.com

Copyright: (c) 2018. Grin-Yatsenko et al. This is an Open Access article distributed under the terms of the Creative Commons Attribution License (CC-BY).

\section{Edited by:}

Rex L. Cannon, PhD, Knoxville Neurofeedback Group, Knoxville, Tennessee, USA

Reviewed by:

Rex L. Cannon, PhD, Knoxville Neurofeedback Group, Knoxville, Tennessee, USA

Randall Lyle, PhD, Mount Mercy University, Cedar Rapids, lowa, USA

\section{Introduction}

Depression is one of the most widespread mental health concerns, causing significant personal distress. The condition does not spontaneously remit in many individuals, and treatment is required for a return to an acceptable quality of life and ability to work. The clinical picture of depression is characterized by distinct emotional symptoms as well as by alterations of cognitive functions such as concentration, memory, and executive control (Austin, Mitchell, \& Goodwin, 2001; Porter, Gallagher, Thompson, \& Young, 2003).

Electrophysiological studies indicate abnormalities in spontaneous electroencephalogram (EEG) in depressed subjects as compared to healthy peers.
The most consistent EEG findings are asymmetry in the alpha band expressed in the increased alpha power in the left frontal region (Davidson, 1995; Davidson \& Henriques, 2000; Davidson et al., 2002; Tomarken \& Keener, 1998) and/or in the right parieto-temporal region (Allen, lacono, Depue, \& Arbisi, 1993; Bruder et al., 1997; Henriques \& Davidson, 1990), bilaterally increased frontal alpha (Brenner et al., 1986; John, Prichep, Fridman, \& Easton, 1988; Lieber \& Newbury, 1988; Pollock \& Schneider, 1990). The inverse relation between alpha power in EEG and cortical activation (Cook, O'Hara, Uijtdehaage, Mandelkern, \& Leuchter, 1998) can reflect cortical hypoactivation in these areas of the brain. Several studies reported an increase in slow-wave activity over the right (Kwon, Youn, \& Jung, 1996; Volf \& Passynkova, 2002) and left 
(Roemer, Shagass, Dubin, Jaffe, \& Siegal, 1992) hemispheres.

Elevated power in alpha, theta, and beta bands in posterior cortical areas was observed in our studies (Grin-Yatsenko, Baas, Ponomarev, \& Kropotov, $2009,2010)$. Increased theta power has been found in depressed patients in several studies (for review see Olbrich \& Arns, 2013). A widespread scalp distribution of theta has been associated with decreased alertness and impaired information processing (Schacter, 1977). The few studies that have investigated theta in major depressive disorder (MDD) using source-localized theta have found this increased theta to be localized to the anterior cingulate cortex (ACC; Jaworska, Blier, Fusee, \& Knott, 2012; Korb, Cook, Hunter, \& Leuchter, 2008). The results of Pizzagalli, Oakes, and Davidson's study (2003) revealed a link between theta and cerebral metabolism in the ACC as well as disruption of functional connectivity within fronto-cingulate pathways in depression. Association of the increased widespread frontal theta with nonresponse to antidepressant treatment was reported in several studies (Arns, Drinkenburg, Fitzgerald, \& Kenemans, 2012; losifescu et al., 2009; Knott, Telner, Lapierre, Browne, \& Horn, 1996; Suffin \& Emory, 1995). High frontal and rostral anterior cingulate theta was found in depressed patients in a study by Arns and colleagues (2016) and associated with treatment nonresponse.

EEG biofeedback (neurofeedback) has been found to be effective in modifying brain function and producing significant improvements in the clinical picture of depressive patients (Baehr, Rosenfeld, \& Baehr, 2001; Hammond, 2000; Othmer, 1994; Rosenfeld, Baehr, Baehr, Gotlib, \& Ranganath, 1996). The Othmer approach was an evolution of the original Sterman protocol for seizure management, which consisted of reinforcement of narrow-band EEG activity in the low beta band. Sterman had settled on $12-15 \mathrm{~Hz}$ as a standard approach (Sterman \& Friar, 1972). This was referred to as the sensorimotor rhythm (SMR). Work with traumatic brain injury by Ayers (1987) led to the adoption of $15-18 \mathrm{~Hz}$ reinforcement (beta1) as a standard training band by Othmers as well, and recovery from the associated depression was routinely reported. Othmers then coupled lefthemisphere training in the beta1 band with SMR training of the right sensorimotor strip in order to address both the left and right hemisphere aspects of the depressive syndrome. By 1997 the training had moved off the sensorimotor strip to engage the prefrontal region with $\mathrm{Fp} 1-\mathrm{C} 3$ for improved activation, and the parietal region with $\mathrm{C} 4-\mathrm{Pz}$ for right-hemisphere overarousal (Othmer, Othmer, \& Kaiser, 1999).

In the early years of this century the training frequencies started to be optimized for each client for improved training efficiency, and this led to a progression to ever lower target frequencies in order to meet the needs of the most challenging clients. Eventually this progression led to the adoption of training in the slow cortical potential (SCP) domain (i.e., below $0.1 \mathrm{~Hz}$ ). This was referred to as infra-low frequency (ILF) training because it was still found to be highly frequency-specific. In this region one had direct access to a measure of cortical activation. That is to say, whereas the SCP reflects various physiological influences, the short-term dynamics of the signal appear to track the dynamics of cortical activation. By using bipolar montage, the measurement is sensitive to the differential cortical activation at the two sites.

The ILF training introduces some novelty into the standard training approach. The feedback to the client is based on a band-limited signal at frequencies below $0.1 \mathrm{~Hz}$, the ILF region. Hence the signal is slowly varying, and thus potentially boring to the trainee. Moreover, the signal level on the screen is arbitrary; the sign of the signal is arbitrary; and the scale factor with which the signal is presented is arbitrary. In consequence, feedback options are provided in which the feedback signal is imbedded in visual imagery of greater visual interest. Thus, the feedback signal is likely to be covert in the perspective of the client. This means that the trainee is not able to volitionally follow the feedback signal. This in turn implies that feedback is contingent on detection of the relevance of the feedback signal to internal state. Trainees typically respond to the signal in a matter of minutes. This becomes apparent through the induction of state shifts-in arousal, vigilance, alertness, and in an emotional state.

This novel approach to training raises a number of questions that have been dealt with at length elsewhere. The question of the frequency-specificity was first treated theoretically in 2008 (Othmer, 2008). The question of how the brain engages with such slow signals is discussed at length in a more recent publication (Othmer \& Othmer, 2017). The mechanisms of recovery are deemed to involve primarily induced alterations of both steady-state and dynamic functional connectivity (Othmer, Othmer, Kaiser, \& Putnam, 2013). Evidence for this 
proposition has recently been furnished through research performed at the Russian Ministry of Health in Moscow. Functional magnetic resonance imaging (fMRI) data were obtained on a number of trainees after single sessions of ILF neurofeedback using procedures identical to those at issue here. Some systematic changes in functional connectivity within the default mode were observed (Dobrushina, 2015).

The first publication on the new method was an observational study on the effect of the ILF training on chronic pain, in particular Complex Regional Pain Syndrome (CRPS1; Jensen, Grierson, Tracy-Smith, Bacigalupi, \& Othmer, 2007). The second was a case series on combat-related PTSD (Othmer \& Othmer, 2009). The third was a case series on pediatric epilepsy (Legarda, McMahon, Othmer, \& Othmer, 2011). A fourth publication considered the application of the method to pediatric neurology generally (Othmer, Othmer, \& Legarda, 2011). Finally, the method is placed in its historical context, and described in considerable detail, in a more recent publication (Othmer et al., 2013). Clinical data are shown for recovery from attentional deficits in a large cohort.

The goal of this study was to assess objectively the efficacy of infra-low frequency neurofeedback by comparing qEEG data before and after 20 sessions in depressed subjects, along with the assessment of the treatment results using three Depression Rating Scales. The working hypothesis is that the improvement of symptoms of depression correlates with decrease of alpha or theta power in EEG in the resting state and task conditions. The follow-up part of our study consisted of the assessment of the qEEG data and the Depression Rating Scales parameters in one year after the first, pretreatment examination.

\section{Methods}

\section{Participants}

Three depressed individuals participated in our study.

Case A. A 43-year-old male suffered from anxiety, depression, chronic fatigue, concentration and memory problems, chronic headache, and joint pain. He has a history of early childhood psychological trauma and difficulties with social communications. This could also be a case of high-functioning autism, but he was never examined to establish that diagnosis.
Case B. A 50-year-old male suffered from depression, loss of interest and motivation to engage in any activity, chronic fatigue, unexplained mood swings during the day, anxiety, tendency to hypercontrol, concentration and memory problems, learning difficulties, alcohol addiction, and sleep problems. He has a history of prenatal stress and childhood psychological trauma (he was brought up without a father)

Case C. A 35-year-old female with chronic fatigue and depression, problems with volitional regulation, the tendency of procrastination, and perfectionism. She also suffers from concentration and working memory problems for the last several years, as well as premenstrual syndrome (PMS). She has a history of early childhood psychological trauma and difficulties with social communication in childhood.

Patients A and B have suffered from depressive complaints for decades; patient $C$ only for the last 6 months. None of the patients sought out doctors for their condition, nor had they ever taken antidepressants.

The baseline investigation consisted of symptom profiles, Depression Rating Scales: MontgomeryÅsberg Depression Rating Scale (MADRS), Hamilton Depression Rating Scale (HAMD), and Beck Depression Inventory (BDI); qEEG in eyesclosed, eyes-open conditions, and in visual cued Go/NoGo test. This took place 1-7 days before undertaking the course of NF training sessions. qEEG parameters were compared with the HBI normative Database. All the tests were repeated after 20 sessions in 1-7 days after the last session, and then again in one year after the first testing. The results of the second and third testing were compared with the pretreatment baseline.

The investigation was carried out in accordance with the declaration of Helsinki and has been approved by the ethics committee of the Institute of Human Brain of the Russian Academy of Sciences, Saint Petersburg. All participants gave written informed consent prior the experiments.

\section{EEG Investigation}

EEG was recorded using a Mitsar 21-channel EEG system (Mitsar, Ltd, St. Petersburg, Russia). Nineteen silver-chloride electrodes were applied according to the International 10-20 system. The input signals referenced to linked ears were filtered between 0.5 and $50 \mathrm{~Hz}$ and digitized at a rate of 250 $\mathrm{Hz}$. The ground electrode was placed on the forehead. All electrode impedances were kept 
below $5 \mathrm{k} \Omega$. EEG was recorded in eyes-closed and eyes-open resting conditions, at least three minutes for every period, and during performance of the visual cued Go/NoGo task that uses pictures of 20 different animals, 20 different plants, and 20 different humans (together with a distracting beep tone) as stimuli (Kropotov, 2009). One trial consisted of the sequential presentation of two pictures (prime and target), presented for $100 \mathrm{~ms}$ each, with an ISI of $1000 \mathrm{~ms}(\mathrm{SOA}=1100 \mathrm{~ms})$. Trials were separated by $1500 \mathrm{~ms}$. Patients were instructed to press the left button of the computer mouse as quickly as possible when an animal was followed by an animal (Go condition), and not to respond when an animal was followed by a plant (NoGo condition), or when a plant was followed by a plant or a human (distractor condition). The response interval lasted from 100 to $1000 \mathrm{~ms}$. The task consisted of $100 \mathrm{Go}$ trials, 100 NoGo trials, and 200 distractor trials. Trials were presented pseudo-randomly with equal probability. All trials were presented to the subject on a computer screen $1.5 \mathrm{~m}$ in front of them using the PsyTask software (Mitsar Ltd., St. Petersburg, Russia). The centrally presented stimuli subtended an approximate visual angle of $3^{\circ}$. Trials with omission and commission errors were excluded from analysis. Quantitative data were obtained using WinEEG software. The epochs with excessive amplitude of nonfiltered EEG and/or excessive high and slow frequency activity were automatically marked and excluded from further analysis.

\section{Neurofeedback}

The instrument used for the clinical neurofeedback was the Cygnet system (BEE Medic), consisting of the NeuroAmp II and Cygnet software, integrated with Somatic Vision video feedback and run on a Windows 7 operating system using a standard personal computer (PC) with a high-resolution monitor. The optimal reinforcement frequency (ORF) was determined for each subject at the beginning of each neurofeedback session on the basis of subjective patient's report. Training was performed with bipolar placement of silver/silver chloride scalp electrodes applied using Ten20 Conductive electrode paste at T4-P4 and T4-Fp2 (according to standard 10-20 system) during the first several sessions, with subsequent adding of T4-T3 and T3-Fp1 locations. The "ground" electrode was placed at Fpz. Each patient received 20 separate 30 - to 45-minute neurofeedback sessions over a period of 7-8 weeks. For each subject the frequency in the infra-slow band was selected individually with bipolar recordings at P4-T4, T4-Fp2, T3-T4, and T3-Fp1. The localization of electrodes was based on the neurofeedback approach developed by Susan Othmer (2017), which involves parameter optimization based on the clinical response.

\section{Statistical Analysis}

The Student criterion was used to estimate statistical significance of differences of EEG spectra values when comparing individual data with the HBI Normative Database and the dynamics of individual EEG spectral parameters after and before treatment. This analysis was performed for each condition (EO, $E C$, and the Go/NoGo task) separately and was carried out for total signal power in five frequency bands: $\delta(1.5-4.0 \mathrm{~Hz})$, theta $(4-8 \mathrm{~Hz}), \alpha(8-12 \mathrm{~Hz})$, $\beta 1 \quad(12-20 \mathrm{~Hz})$ and $\beta 2(20-30 \mathrm{~Hz})$, that was computed as a sum of power for corresponding frequencies.

\section{Results}

\section{Assessment Before Initiating Neurofeedback}

Case A. Depression scales and inventory. MADRS rating $=26$. $\mathrm{BDI}$ rating $=16$. HAMD rating $=21$. In sum the Depression scales ratings indicated moderate degree of depression.

EEG investigation (as compared to the $\mathrm{HBI}$ Normative Database). Eyes-open state: elevated absolute theta activity of $6-8 \mathrm{~Hz}$ over midline frontalcentral area $(\mathrm{Fz}, \mathrm{Cz})(t=2.23, p<.05)$ and relative beta2 activity over the parietal and posterior temporal area $(t=-2.94, p<.01)$; increased relative a power $(8-9.5 \mathrm{~Hz})$ over occipital, parietal, and posterior temporal cortical areas with maximal difference at P4 $(t=2.66, p<.01)$.

Eyes-closed state: increased relative slow a activity of $7.5-8.5 \mathrm{~Hz}$ over occipital, parietal, and posterior temporal areas $(t=2.58, p<.05)$ and relative beta2 activity over frontal-central area $(t=3.47, p<.001)$.

Go/NoGo task: increased absolute theta activity of $5.5-7 \mathrm{~Hz}$ over frontal area $(t=2.04, p<.05)$, and absolute beta2 activity of $25-28 \mathrm{~Hz}$ over midline frontal area (Fz; $t=2.38, p<.05)$. Slow $a$ rhythm spread over anterior cortical areas, relative slow $\alpha$ power $(7.5-9.5 \mathrm{~Hz})$ is increased over occipital, parietal, and posterior temporal cortical areas with maximum at $\mathrm{P} 3(t=2.65, p<.01)$.

The increased level of theta and slow $\alpha$ activity over the frontal area might be a correlate of poor concentration, and with depressive symptoms. Enhanced high beta power over frontal-central region can be a sign of anxiety. 
Case B. Depression scales and inventory. MADRS rating $=20$. $\mathrm{BDI}$ rating $=26$. HAMD rating $=16$. In sum the Depression scales ratings indicated mild to moderate degree of depression.

EEG investigation (as compared to the $\mathrm{HBI}$ Normative Database). Eyes-open state: increased relative a power of $9.5-10.5 \mathrm{~Hz}$ over parietal, central and frontal areas, maximal at $\mathrm{Cz}(t=2.54, p<.05)$. Episodic groups of $\alpha$ and theta waves arise at temporal sites of both left and right hemispheres.

Eyes-closed state: a rhythm of $10 \mathrm{~Hz}$ spread over frontal cortical areas, episodes of $\alpha$ and theta waves at temporal sites of both left and right hemispheres.

Go/NoGo task: Increased relative a power of 9.5$11 \mathrm{~Hz}$ over temporal, central and frontal areas, maximal at $\mathrm{Cz}(t=-2.46, p<.05)$.

The increased level of $\alpha$ activity over frontal area, and increased $\alpha$ and theta activity over temporal areas might be a marker of chronic stress, depression, fatigue, and poor concentration.

Case C. Depression scales and inventory. MADRS rating $=17$. BDI rating $=18$. HAMD rating $=15$. In sum the Depression scales ratings indicated mild to moderate degree of depression.

EEG investigation (as compared to the $\mathrm{HBI}$ Normative Database). Eyes-open state: episodes of spreading of a rhythm over frontal cortical areas. Increased absolute beta2 $(t=-3.70, p<.001)$ and increased relative theta $(t=-5.63, p<.0001), \alpha(t=$ $-3.82, p<.001)$ and beta2 $(t=-3.44, p<.001)$ activity over frontal areas.

Eyes-closed state: increased relative theta power over parietal $(t=-4.78, p<.0001)$ and left posterior temporal area $(t=-2.99, p<.001)$; prevalence of $\alpha$ rhythm of $11 \mathrm{~Hz}$ over the left parietal-occipital area, spreading of $\alpha$ rhythm over frontal cortical areas.
Go/NoGo task: Increasing of both absolute $(t=$ $-2.79, p<.01)$ and relative $(t=-2.91, p<.01)$ beta2 activity over frontal and central area.

The spreading of a activity over the frontal area might be a correlate of poor concentration, volitional regulation and working memory, and also with depressive complaints. Increased beta activity and high-frequency basic rhythm can be the signs of high vigilance and perfectionism.

\section{Posttreatment Assessment}

Case A. After completion of 20 neurofeedback (NFB) sessions, the patient indicated disappearance of tension in his body and an increase of energy level. He has improved the perception of his body and the surrounding space. His headaches became less intense and arose more seldom. He reported on improvement of concentration and stable positive mood from the middle of the training course.

Control assessment. The Depression profile score during the second testing was: MADRS rating $=2$ (improvement of $92.3 \%$ ). BDI rating $=1$ (improvement of 93.75\%). HAMD rating $=2$ (improvement of 90.5\%). After NFB course the Depression scales ratings indicated no signs of depression.

EEG in eyes-open condition (Figure 1) showed significant decrease of both theta $(t=12.80, p$ $<.0001)$ and beta2 $(t=5.88, p<.0001)$ activity at frontal and central sites; during Go/NoGo task performance decreasing of absolute theta $(t=5.68$, $p<.0001)$, and beta2 $(t=288.71, p<.001)$ power was also most prominent over frontal and central cortical areas (Figure 3). The absolute a rhythm power decreased significantly at all sites mostly in eyes-open condition with maximal differences at P4 $(t=-6.60, p<.0001)$, which reflects the higher cortical activation. In eyes-closed state a power increased in parietal cortical areas (Figure 2), which might relate to a better ability to relax. 


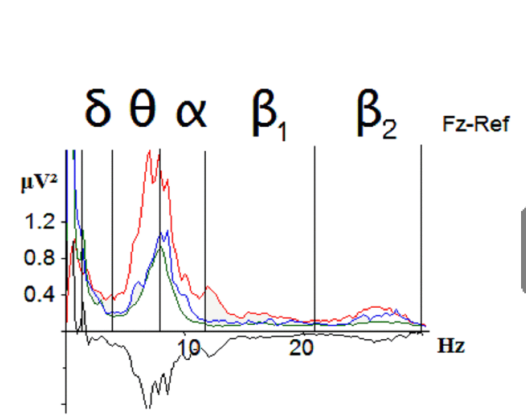

\section{Case A}
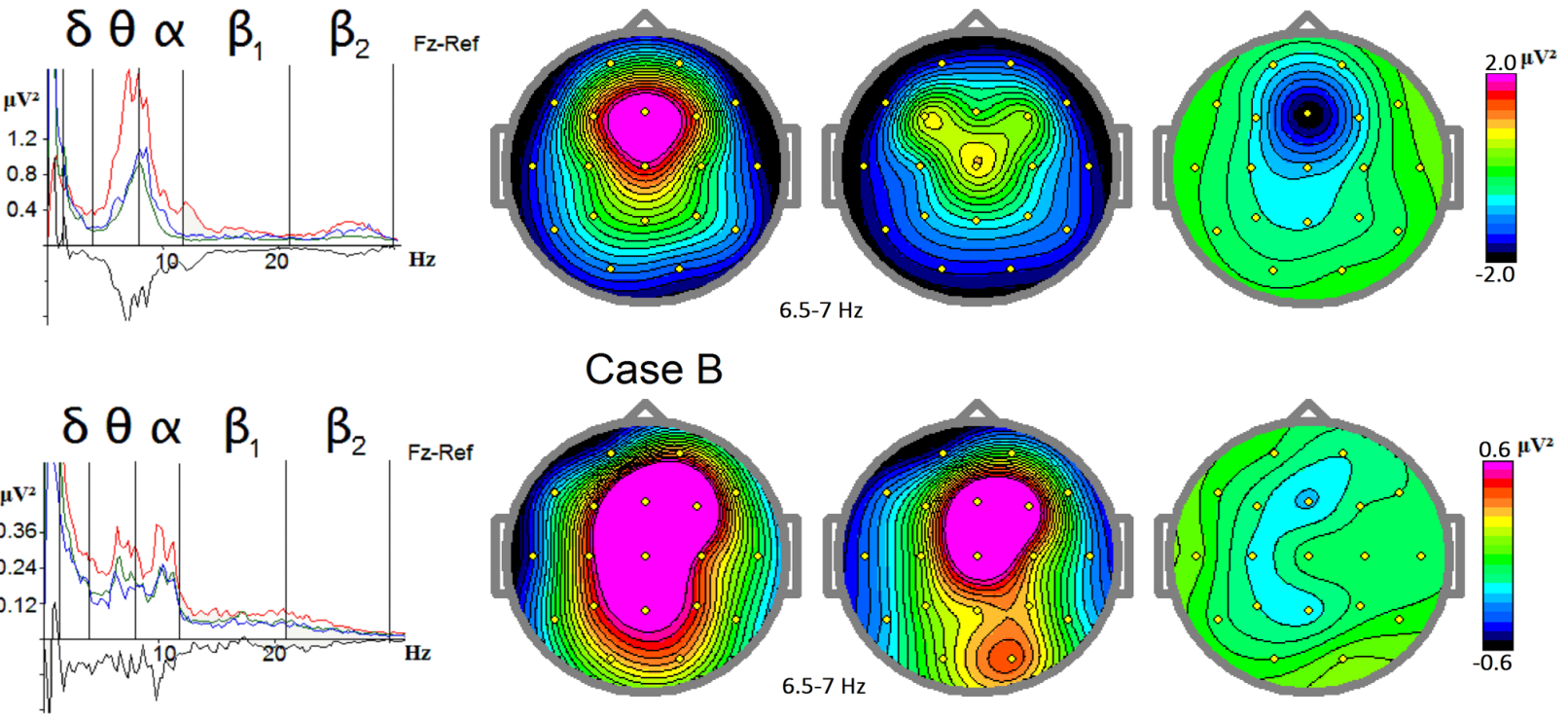

Case C
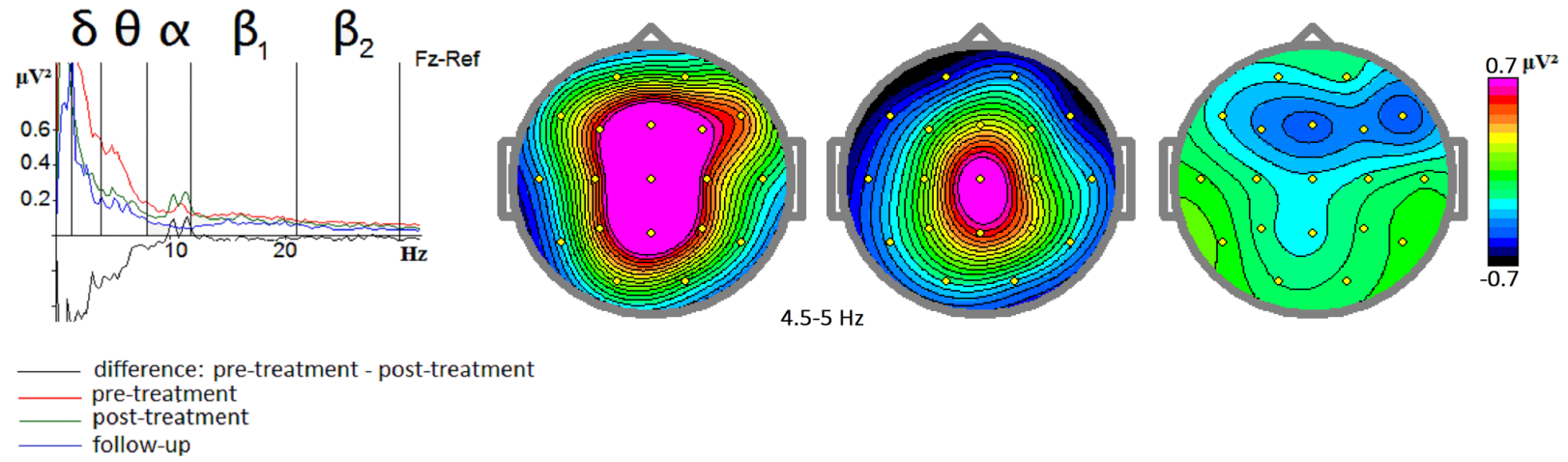

\section{a}

b

C

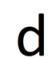

Figure 1. Dynamics of EEG spectra after ILF NF course in depressed patients in eyes-open condition. Grand average power spectra of the raw EEG in patients $A, B$, and C are presented in (a). Xaxis $=$ frequency in $\mathrm{Hz}, \mathrm{Y}$ axis $=$ power in $\mu \mathrm{V}^{2}$. Maps of theta power in the selected frequency band (indicated below the maps) in pretreatment and posttreatment EEGs are represented in (b) and (c) correspondingly. Maps of difference of theta power in the selected frequency between posttreatment and pretreatment EEGs are shown in (d). Scale is presented at right of the maps.

\section{Case B.}

After completion of 20 NFB sessions, the patient reported a dramatic decrease of inner tension and reactivity to stressful situations. His emotional stability increased, and the level of anxiety diminished. Relationship with other people improved; he began to understand them better. He has significantly reduced the use of alcohol. $\mathrm{He}$ reported that confidence, calmness, and a sense of power had returned to him. The depressive mood disappeared, and he felt clarity in his mind and had constructive ideas on the organization of his future life.

Control assessment. The Depression profile score during the second testing was: MADRS rating $=6$ (improvement of 70\%). BDI rating $=2$ (improvement of $92.3 \%$ ). HAMD rating $=5$ (improvement of $68.75 \%)$. After NFB course the Depression scales ratings indicated no signs of depression. 
EEG showed a significant decreasing of power of both alpha $(t=7.97, p<.0001)$ and theta $(t=48.74$, $p<.0001)$ activity over frontal area in eyes-open state (Figure 1), and decreasing of theta power over frontal, central and parietal area during Go/NoGo task performance (Figure 3) with maximal changes at $\mathrm{Fz}(t=2.39, p<.05)$, which might be a result of better activation of frontal cortex. In eyes-closed state the maximum of alpha rhythm power shifted from the parietal to the occipital area (Figure 2), which is a normal distribution of the alpha rhythm with eyes closed.

\section{Case A}
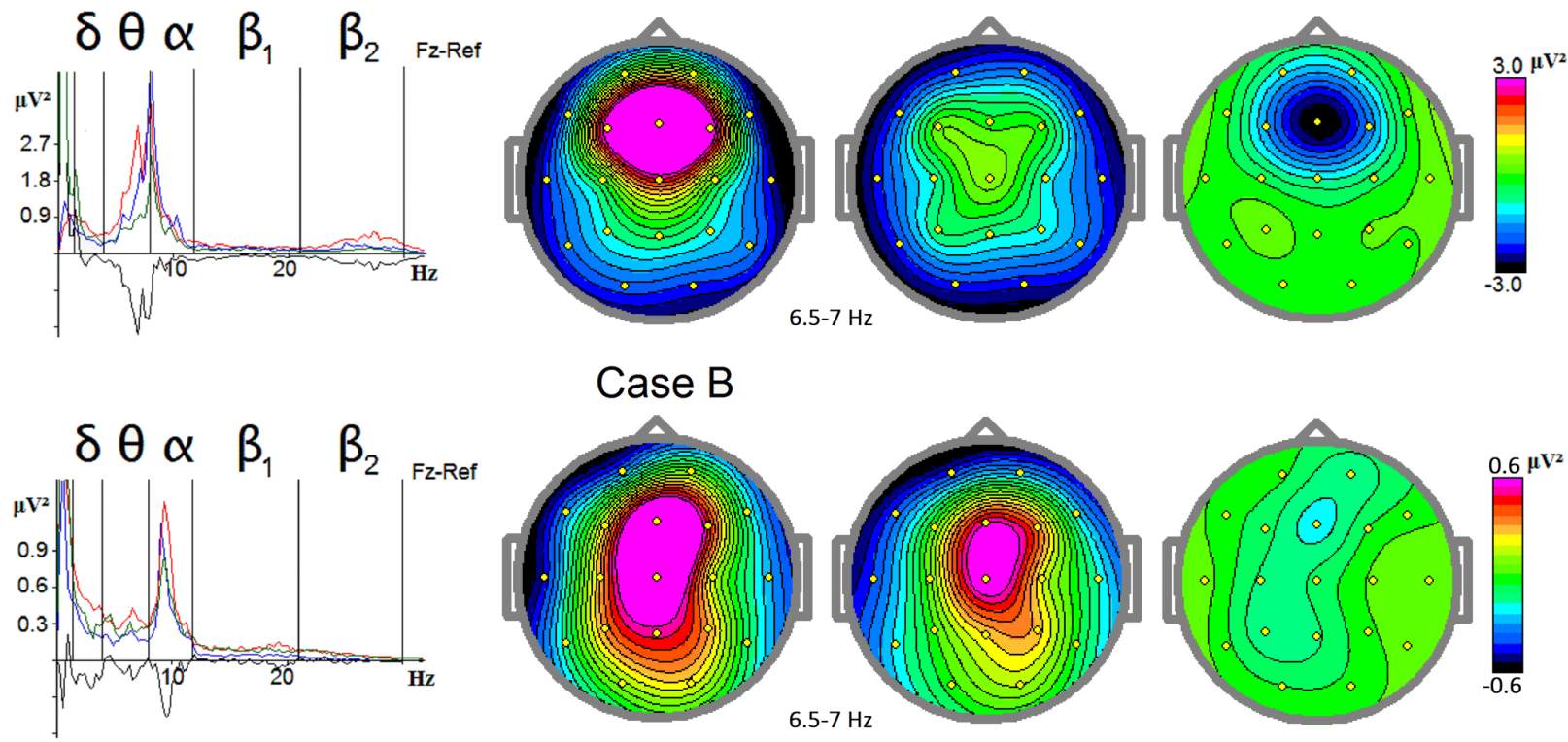

\section{Case C}
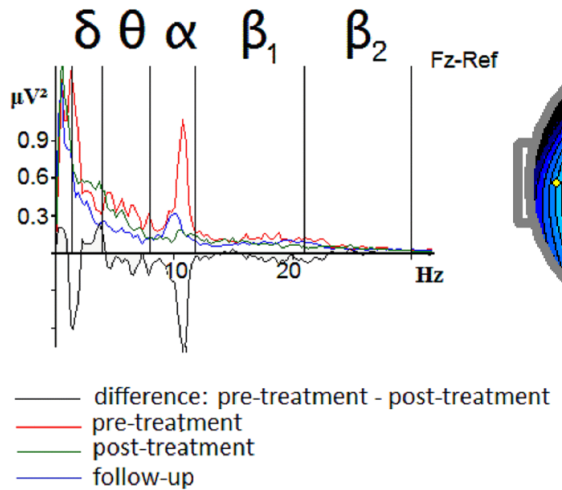

a

b

C

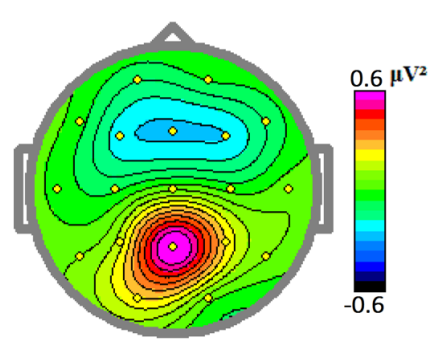

Figure 2. Dynamics of EEG spectra after ILF NF course in depressed patients in eyes-closed condition. Grand average power spectra of the raw EEG in patients $A, B$, and $C$ are presented in (a). Xaxis $=$ frequency in $\mathrm{Hz}, \mathrm{Y}$ axis $=$ power in $\mu \mathrm{V}^{2}$. Maps of theta power in the selected frequency band (indicated below the maps) in pretreatment and posttreatment EEGs are represented in (b) and (c) correspondingly. Maps of difference of theta power in the selected frequency between posttreatment and pretreatment EEGs are shown in (d). Scale is presented at right of the maps. 
Case C. After completion of 20 NFB sessions, the patient reported remarkable improvement of her mood and decrease of anxiety level. She became calmer, more able to deal with sudden unplanned and stressful events. She developed the intention to act to achieve new goals and a clear understanding of what activities are necessary and valuable, and which are not. She started to exercise regularly; her mental performance and success at work increased remarkably.

Control assessment. The Depression profile score during the second testing was: MADRS rating $=4$ (improvement of $76.5 \%$ ). BDI rating $=3$ (improvement of $83.3 \%$ ). HAMD rating $=3$ (improvement of $81.4 \%$ ). After NFB course the Depression scales ratings indicated no signs of depression.

EEG showed a significant decreasing of power of theta activity $(t=3.82, p<.001)$ at frontal and central sites in eyes-open condition (Figure 1 ), and decreasing of theta power over frontal and central sites during Go/NoGo task $(t=11.45, p<.0001)$, which might be a correlate of the higher cortical functional activity in this region (Figure 3 ).
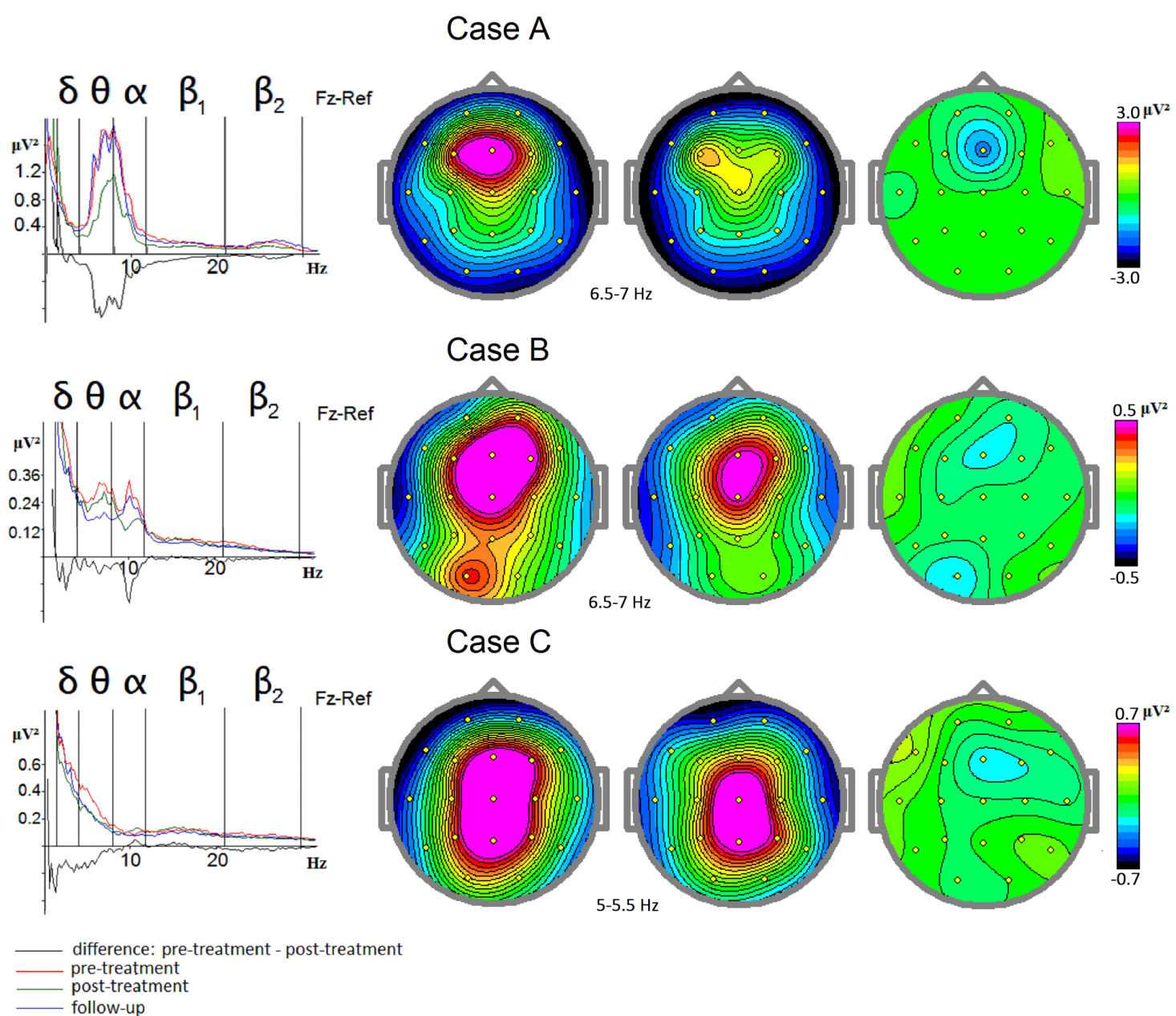

b

C

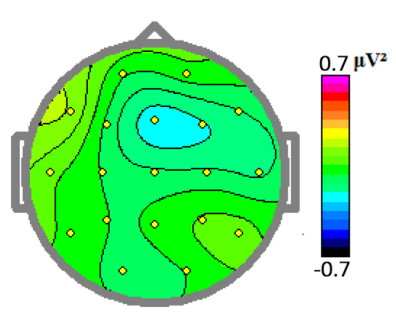

a

difference: pre-treatment - post-treatment
pre-treatment
post-treatment
follow-up

Case C

Figure 3. Dynamics of EEG spectra after ILF NF course in depressed patients during Go/NoGo task performance. Grand average power spectra of the raw EEG in patients A, B, and C are presented in (a). $\mathrm{X}$-axis $=$ frequency in $\mathrm{Hz}, \mathrm{Y}$ axis = power in $\mu \mathrm{V}^{2}$. Maps of theta power in the selected frequency band (indicated below the maps) in pretreatment and posttreatment EEGs are represented in (b) and (c) correspondingly. Maps of difference of theta power in the selected frequency between posttreatment and pretreatment EEGs are shown in (d). Scale is presented at right of the maps. 


\section{Follow-up Assessment}

Case A. One year after the start of the ILF NF course, the patient reported that all positive changes in his state remained despite the fact that he has been overloaded by work. He perceives his thoughts, emotions, and moods mindfully, and he is able to manage them. The headaches arise very seldom, and his improved concentration has maintained.

Control assessment (as compared to the pretreatment assessment). The Depression profile score during the second testing was: MADRS rating $=0$ (improvement of $100 \%$ ). BDI rating $=1$ (improvement of $94 \%$ ). HAMD rating $=0$ (improvement of 100\%). The Depression scales ratings indicated no signs of depression, and the scores improved as compared to in posttreatment assessment.

EEG. Decrease of theta activity at frontal and central sites observed after treatment remains in the third EEG in eyes-open (Figure 1) and eyes-closed (Figure 2) state. However, during Go/NoGo task performance theta power at anterior sites returned to the pretreatment level (Figure 3). Alpha power decreased at central and parietal sites in all states, and increased at occipital sites in the last EEG, which corresponds to the normal alpha rhythm distribution.

Case B. One year after the pretreatment investigation, the patient reported that improvement of his mood after ILF NF remained steady. He does not suffer from the mood swings any more, and depressive and unsettling thoughts arise quite rarely. He seldom uses alcohol, sleeps well, his emotional stability and level of energy are high, and he experiences "lust for life." Yet there remain the concentration problems, and his memory is still poor.

Control assessment (as compared to the pretreatment assessment). The Depression profile score during the second testing was: MADRS rating $=5$ (improvement of 75\%). BDI rating $=9$ (improvement of $69.3 \%$ ). HAMD rating $=3$ (improvement of $81.4 \%$ ). The Depression scales ratings indicated no signs of depression, and the scores even improved as compared to the posttreatment assessment for two of the three scales.

EEG. Decreasing of theta and alpha activity at frontal sites observed after treatment remains in the third EEG in eyes-open (Figure 1) and eyes-closed (Figure 2) states, and during Go/NoGo test (Figure
3). Frontal theta power during Go/NoGo task is lower than in posttreatment EEG. There remains a decrease of alpha power (as compared to the pretreatment EEG) in parietal region and alpha prevalence in occipital area in eyes-closed state.

Case C. One year after the first (pretreatment) investigation, the patient reports that the positive shift in her mood obtained after the course of treatment remains stable. She does not suffer from depression or fatigue since the training finished. At the same time, she sometimes experiences procrastination and insufficient concentration.

Control assessment (as compared to the pretreatment assessment). The Depression profile score during the second testing was: MADRS rating $=4$ (improvement of $76.5 \%$ ). $\mathrm{BDI}$ rating $=5$ (improvement of $72.2 \%$ ). HAMD rating $=3$ (improvement of $80 \%$ ). The Depression scales ratings indicated no signs of depression, and the scores are about the same level as in posttreatment assessment.

EEG. The decrease of theta activity at frontal and central sites in eyes-open condition (Figure 1) and during Go/NoGo test performance (Figure 3) observed after treatment remains in the third EEG. Alpha power decreased at all sites in eyes-open state and during Go/NoGo test. In eyes-closed state (Figure 2) the initially excessive alpha power decreased all over the scalp, but predominantly in parietal and occipital cortical areas of the left hemisphere.

\section{Discussion}

In our study, we selected a protocol that implemented infra-slow EEG frequencies (below 0.1 $\mathrm{Hz}$ ) as a biofeedback parameter in three depressed individuals. The time course of the signal at these very slow frequencies reflects variations in cortical excitability (Vanhatalo et al., 2004; van Putten, Tjepkema-Cloostermans, \& Hofmeijer, 2014). This suggests that the most direct effect of the neurofeedback challenge is on the regulation of tonic central arousal. The within-session effects of perceived calming are consistent with this hypothesis.

The choice of principal electrode placements was driven originally by considerations of functional neuroanatomy and was then refined on the basis of empirical observation over the course of many years (Othmer, 2017). The principal sites correspond with the multimodal association areas in cortex, which 
reflect cortical activity at its most integrative stage. These are also the regions most readily subject to dysregulation. Further, these sites correspond to those regions in which the principal hubs of the default mode are accessible to us at the cortical surface (Buckner, Andrews-Hanna, \& Schacter, 2008). Since engagement with the outside world constitutes at most a modest perturbation on baseline activity, as demonstrated by cortical hemodynamics, the burden of functionality is carried mainly by our task-negative network, the default mode, and the training addresses itself to that as a first priority (Raichle, 2011). A secondary objective is to train the salience network, which mediates between the task-negative and the task-positive network, the Central Executive. Menon (2011) has made the case that a number of major psychopathologies can be traced to deficits in the functional connectivities within and among our intrinsic connectivity networks. Broyd et al. (2009) have evaluated the role of the default mode in mental disorders more inclusively.

On the basis of the Menon model, the favorable outcomes in this study may be attributed at least partly to the renormalization of functional connectivity within the default mode, and to altered relationship between the default mode, the Salience Network, and the Central Executive. That in turn would imply improved functionally specific activation, which could also account for the remediation of the depressive state. This hypothesis remains to be proved through independent measurement.

For the above reasons, our approach differs from the conventional protocols for the treatment of depression that are based on models of altered hemispheric asymmetry, and use either an alpha asymmetry protocol (Baehr \& Baehr, 1997; Baehr et al., 2001; Baehr, Rosefeld, Baehr, \& Earnest, 1998, 1999; Choi et al., 2011; Dias \& Van Deusen, 2011; Earnest, 1999; Rosenfeld, 2000; Rosenfeld et al., 1996) or a relative left frontal beta enhancement with concomitant theta suppression (Othmer et al., 1999), or a theta/beta ratio reduction (Dias \& Van Deusen, 2011). However, as the results of the present study indicate, the application of our protocol turns out to be as effective as conventional protocols.

In each of three patients 20 sessions of this type of neurofeedback considerably improved mood and self-organization skills, decreased anxiety and inner tension, and increased emotional stability and stress tolerance. Their clinical symptoms, as assessed with the Depression Rating Scales: MADRS, HAMD and $\mathrm{BDI}$, improved significantly and did not indicate depression any more. Moreover, the improvements of the Depression profile scores obtained after ILF NF training remained stable over one year after the beginning of ILF NF course in all three patients.

Pretreatment qEEG investigation in two participants of our study revealed absolute and/or relative theta, alpha, and beta2 elevation, as compared to the normative database, in passive conditions and during Go/NoGo task performance. The differences were observed mostly in frontal and central areas of the brain. These findings are in agreement with studies that found abnormally large theta (Jaworska et al., 2012; Korb et al., 2008; Strijkstra at al., 2003) and alpha (Lieber \& Newbury, 1988; Pollock \& Schneider, 1990) power in depressed patients in anterior regions, which may relate to diminished frontal cortical activation (Cook et al. 1998; Strijkstra at al., 2003). Enhanced beta activity is also reported in depressed patients (Fingelkurts et al., 2006; GrinYatsenko et al., 2009, 2010; Shankman \& Klein, 2003; Yamada, Kimura, Mori, \& Endo, 1995). This phenomenon was observed in the EEG when anxiety symptoms were part of the clinical picture. In one patient, pretreatment theta and beta were borderline normal, but relative frontal and central alpha activity was elevated.

Our study showed that ILF NF sessions led to a significant decrease of theta power over frontal and central areas in all three patients in passive states and during Go/NoGo task performance. Besides, frontal alpha and beta decreased in those patients in whom the pretreatment powers in these bands were enhanced. These EEG spectral dynamics might be a correlate of ILF NF related recovery of the level of frontal cortical activation.

The results of this research are comparable to the data of previously reported studies (Baehr, Rosefeld, \& Baehr, 1998; Dias \& Van Deusen, 2011; Earnest, 1999; Rosenfeld et al., 1996), which used neurofeedback based on EEG spectra in depression. Recent studies report on the role of infra-slow cortical potential oscillations in the modulation of conventional frequency bands (Lörincz, Geall, Bao, Crunelli, \& Hughes, 2009; van Putten et al., 2015). Therefore, modulation of these infra-slow oscillations during ILF NF sessions could exert a modulating and normalizing influence on EEG bands in depressed individuals participated in our study. Conversely, conventional band training could derive its effectiveness at least partially from the correlation with infra-low frequency activity. Both methods appear successful in mobilizing a system response that gradually allows functional 
renormalization to occur. This may explain the similarity of positive results in treatment of depression in our study and in the works of researchers who used the conventional EEG spectra neurofeedback in treatment of this disorder.

The follow-up qEEG data showed that decreased frontal theta power remained stable in two patients. Still in one patient theta went up almost to the pretreatment level during the $\mathrm{Go} / \mathrm{NoGo}$ task challenge, despite the fact that his clinical achievements were sustained, and baseline EEG measures were maintained in their posttraining status. This observation could indicate some ambiguity in the relationship of changes in the level of theta activity and the efficiency of functioning of the affective network. The elevated theta activity could simply be the result of increased cognitive demand. Probably the interrelation of these characteristics is more complex than a direct relationship.

The present study reveals qEEG correlates of ILF NF in depression. It shows that not only psychophysical parameters of affective network functioning improve by the neurotherapy, but also objective neurophysiological parameters change, reflecting improvement of emotional stability and control in depressed individuals after ILF NF.

\section{Acknowledgements}

The authors wish to thank Susan Othmer, Clinical Director of the EEG Institute, Woodland Hills, California, for development of neurofeedback protocols used in this study; Dr. Bernhard Wandernoth of BEE Medic, engineer and head of development of the Cygnet neurofeedback system used in this study; Dr. Andreas Müller, Director of the Research Clinic, Chur, Switzerland, for providing us with EEG spectra obtained in healthy subjects.

\section{Conflict of Interest}

The authors declare no conflicts of interest with respect to the research, authorship, and/or publication of this article. The second author (SO) was only involved in a critical review of the manuscript but had no ability to influence the research design, the conduct of the research, or the analysis and presentation of the data.

\section{References}

Allen, J. J., lacono, W. G., Depue, R. A., \& Arbisi, P. (1993). Regional electroencephalographic asymmetries in bipolar seasonal affective disorder before and after exposure to bright light. Biological Psychiatry, 33(8-9), 642-646. http://dx.doi.org/10.1016/0006-3223(93)90104-L
Arns, M., Bruder, G., Hegerl, U., Spooner, C., Palmer, D. M., Etkin, A., \& Gordon, E. (2016). EEG alpha asymmetry as a gender-specific predictor of outcome to acute treatment with different antidepressant medications in the randomized iSPOT-D study. Clinical Neurophysiology, 127(1), 509-519. http://dx.doi.org/10.1016/j.clinph.2015.05.032

Arns, M., Drinkenburg, W. H., Fitzgerald, P. B., \& Kenemans, J. L. (2012). Neurophysiological predictors of non-response to rTMS in depression. Brain Stimulation, 5(4), 569-576. http://dx.doi.org/10.1016/j.brs.2011.12.003

Austin, M.-P., Mitchell, P., \& Goodwin, G. M. (2001). Cognitive deficits in depression: Possible implications for functional neuropathology. The British Journal of Psychiatry, 178(3), 200-206. http://dx.doi.org/10.1192/bjp.178.3.200

Ayers, M. E. (1987). Electroencephalographic neurofeedback and closed head injury of 250 individuals. In National Head Injury Syllabus (pp. 380-392). Washington, DC: Head Injury Foundation.

Baehr, E., \& Baehr, R. (1997). The use of neurofeedback as adjunctive therapeutic treatment for depression: Three case studies. Biofeedback, 25(1), 10-11.

Baehr, E., Rosenfeld, J. P., \& Baehr, R. (1998). The clinical use of an alpha asymmetry biofeedback protocol in treatment of depressive disorders: Two case studies. Journal of Neurotherapy, 2, 12-27.

Baehr, E., Rosenfeld, J. P., \& Baehr, R. (2001). Clinical use of an alpha asymmetry neurofeedback protocol in the treatment of mood disorders: Follow-up study one to five years post therapy. Journal of Neurotherapy, 4(4), 11-18. http://dx.doi.org/10.1300/J184v04n04_03

Baehr, E., Rosenfeld, J. P., Baehr, R., \& Earnest, C. (1998). Comparison of two EEG asymmetry indices in depressed patients vs. normal controls. International Journal of Psychophysiology, 31(1), 89-92. http://dx.doi.org/10.1016 IS0167-8760(98)00041-5

Baehr, E., Rosenfeld, J. P., Baehr, R., \& Earnest, C. (1999). Clinical use of an alpha asymmetry protocol in the treatment of mood disorders. In J. R. Evans \& A. Abarbanel (Eds.), Introduction to quantitative EEG and neurofeedback (pp.181188). New York, NY: Academic Press. http://dx.doi.org /10.1016/B978-012243790-8/50009-2

Brenner, R. P., Ulrich, R. F., Spiker, D. G., Sclabassi, R. J., Reynolds, C. F., Marin, R. S., \& Boller, F. (1986) Computerized EEG spectral analysis in elderly normal, demented and depressed subjects. Electroencephalography and Clinical Neurophysiology, 64(6), 483-492. http://dx.doi.org/10.1016/0013-4694(86)90184-7

Broyd, S. J., Demanuele, C., Debener, S., Helps, S. K., James, C. J., \& Sonuga-Barke, E. J. S. (2009). Default-mode brain dysfunction in mental disorders: a systematic review. Neuroscience \& Biobehavioral Reviews, 33(3), 279-296. http://dx.doi.org/10.1016/j.neubiorev.2008.09.002

Bruder, G. E., Fong, R., Tenke, C. E., Leite, P., Towey, J. P., Stewart, J. E., \& Quitkin, F. M. (1997). Regional brain asymmetries in major depression with or without an anxiety disorder: A quantitative electroencephalographic study. Biological Psychiatry, 41(9), 939-948. http://dx.doi.org /10.1016/S0006-3223(96)00260-0

Buckner, R. L., Andrews-Hanna, J. R., \& Schacter, D. L. (2008). The brain's default network: anatomy, function, and relevance to disease. Annals of the New York Academy of Sciences, 1124(1), 1-38. http://dx.doi.org/10.1196/annals.1440.011

Choi, S. W., Chi, S. E., Chung, S. Y., Kim, J. W., Ahn, C. Y., \& Kim, H. T. (2011). Is alpha wave neurofeedback effective with randomized clinical trials in depression? A pilot study. Neuropsychobiology, 63(1), 43-51. http://dx.doi.org/10.1159 1000322290

Cook, I. A., O'Hara, R., Uijtdehaage, S. H. J., Mandelkern, M., \& Leuchter, A. F. (1998). Assessing the accuracy of topographic EEG mapping for determining local brain function. 
Electroencephalography and Clinical Neurophysiology, 107(6), 408-414. http://dx.doi.org/10.1016/S00134694(98)00092-3

Davidson, R. J. (1995). Cerebral asymmetry, emotion and affective style. In R. J. Davidson \& K. E. Hugdahl (Eds.), Brain Asymmetry (pp. 107-123). Cambridge, MA: MIT Press.

Davidson, R. J., \& Henriques, J. (2000). Regional brain function in sadness and depression. In J. C. Borod (Ed.), The neuropsychology of emotion. Series in affective science (pp. 269-297). New York, NY: Oxford University Press.

Davidson, R. J., Lewis, D. A., Alloy, L. B., Amaral, D. G., Bush, G., Cohen, J. D., \& Peterson, B. S. (2002). Neural and behavioral substrates of mood and mood regulation. Biological Psychiatry, 52(6), 478-502. http://dx.doi.org /10.1016/S0006-3223(02)01458-0

Dias, Á. M., \& van Deusen, A. (2011). A new neurofeedback protocol for depression. The Spanish Journal of Psychology, 14(01), 374-384. /rev_SJOP.2011.v14.n1.34

Dobrushina, O. R., Vlasova, R., Pechenkova E. V., Rumshiskaya, A. D., Litvinova, L. D., Mershina, E. A., \& Sinitsyn V. E. (2015, May). The effect of Infra-Low Frequency Neurofeedback on default mode network of the brain (in Russian, pp. 27-28). Conference on Applied Neuroscience and Social Well-Being, Moscow, Russia. http://dx.doi.org/10.13140 /RG.2.1.4272.7122

Earnest, C. (1999). Single case study of EEG asymmetry biofeedback for depression: An independent replication in an adolescent. Journal of Neurotherapy, 3(2), 28-35. http://dx.doi.org/10.1300/J184v03n02_04

Fingelkurts, A. A., Fingelkurts, A. A., Rytsälä, H., Suominen, K., Isometsä, E., \& Kähkönen, S. (2006). Composition of brain oscillations in ongoing EEG during major depression disorder. Neuroscience Research, 56(2), 133-144. http://dx.doi.org /10.1016/j.neures.2006.06.006

Grin-Yatsenko, V. A., Baas, I., Ponomarev, V. A., \& Kropotov, J. D. (2009). EEG power spectra at early stages of depressive disorders. Journal of Clinical Neurophysiology, 26(6), 401406. http://dx.doi.org/10.1097/WNP.0b013e3181c298fe

Grin-Yatsenko, V. A., Baas, I., Ponomarev, V. A., \& Kropotov, J. D. (2010). Independent component approach to the analysis of EEG recordings at early stages of depressive disorders. Clinical Neurophysiology, 121(3), 281-289. http://dx.doi.org /10.1016/j.clinph.2009.11.015

Hammond, D. C. (2000). Neurofeedback treatment of depression with the Roshi. Journal of Neurotherapy, 4(2), 45-56. http://dx.doi.org/10.1300/J184v04n02_06

Henriques, J. B., \& Davidson, R. J. (1990). Regional brain electrical asymmetries discriminate between previously depressed and healthy control subjects. Journal of Abnormal Psychology, 99(1), 22-31. http://dx.doi.org/10.1037/0021843X.99.1.22

losifescu, D. V., Greenwald, S., Devlin, P., Mischoulon, D., Denninger, J. W., Alpert, J. E., \& Fava, M. (2009). Frontal EEG predictors of treatment outcome in major depressive disorder. European Neuropsychopharmacology, 19(11), 772777. http://dx.doi.org/10.1016/j.euroneuro.2009.06.001

Jaworska, N., Blier, P., Fusee, W., \& Knott, V. (2012). Alpha power, alpha asymmetry and anterior cingulate cortex activity in depressed males and females. Journal of Psychiatric Research, 46(11), 1483-1491. http://dx.doi.org/10.1016 /j.jpsychires.2012.08.003

Jensen, M. P., Grierson, C., Tracy-Smith, V., Bacigalupi, S. C., \& Othmer, S. (2007). Neurofeedback treatment for pain associated with Complex Regional Pain Syndrome Type I. Journal of Neurotherapy, 11(1), 45-53. http://dx.doi.org /10.1300/J184v11n01_04

John, E. R., Prichep, L. S., Fridman, J., \& Easton, P. (1988). Neurometrics: Computer-assisted differential diagnosis of brain dysfunctions. Science, 239(4836), 162-169. http://dx.doi.org/10.1126/science.3336779

Knott, V. J., Telner, J. I., Lapierre, Y. D., Browne, M., \& Horn, E. R. (1996). Quantitative EEG in the prediction of antidepressant response to imipramine. Journal of Affective Disorders, 39(3), 175-184. http://dx.doi.org/10.1016/01650327(96)00003-1

Korb, A. S., Cook, I. A., Hunter, A. M., \& Leuchter, A. F. (2008). Brain electrical source differences between depressed subjects and healthy controls. Brain Topography, 21(2), 138146. http://dx.doi.org/10.1007/s10548-008-0070-5

Kropotov, J. (2009). Quantitative EEG, event-related potentials and neurotherapy. San Diego, CA: Academic Press/Elsevier.

Kwon, J. S., Youn, T., \& Jung, H. Y. (1996). Right hemisphere abnormalities in major depression: Quantitative electroencephalographic findings before and after treatment. Journal of Affective Disorders, 40(3), 169-173. http://dx.doi.org/10.1016/0165-0327(96)00057-2

Legarda, S. B., McMahon, D., Othmer, S., \& Othmer, S. (2011). Clinical neurofeedback: Case studies, proposed mechanism, and implications for pediatric neurology practice. Journal of Child Neurology, 26(8), 1045-1051. http://dx.doi.org/10.1177 10883073811405052

Lieber, A. L., \& Newbury, N. D. (1988). Diagnosis and subtyping of depressive disorders by quantitative electroencephalography: III. Discriminating unipolar from bipolar depression. Hillside Journal of Clinical Psychiatry, 10(2), 165-172.

Lörincz, M. L., Geall, F., Bao, Y., Crunelli, V., \& Hughes, S. W. (2009). ATP-dependent infra-slow $(<0.1 \mathrm{~Hz})$ oscillations in thalamic networks. PLoS One, 4(2), e4447. http://dx.doi.org/ 10.1371/journal.pone.0004447

Menon, V. (2011). Large-scale brain networks and psychopathology: A unifying triple network model. Trends in Cognitive Sciences, 15(10), 483-506. http://dx.doi.org /10.1016/j.tics.2011.08.003

Olbrich, S., \& Arns, M. (2013). EEG biomarkers in major depressive disorder: Discriminative power and prediction of treatment response. International Review of Psychiatry, 25(5), 604-618. http://dx.doi.org/10.3109/09540261.2013.816269

Othmer, S. (1994). Depression. In Training Syllabus (Vol. II). Encino, CA: EEG Spectrum.

Othmer, S. (2008). Neuromodulation technologies: An attempt at classification. In T. H. Budzynski, H. K. Budzynski, J. R. Evans, \& A. Abarbanel (Eds.), Introduction to Quantitative EEG and Neurofeedback: Advanced Theory and Applications (2nd ed., pp. 3-26). Burlington, MA: Elsevier Academic Press. http://dx.doi.org/10.1016/B978-0-12-374534-7.00001-0

Othmer, S., \& Othmer, S. F. (2009). Post traumatic stress disorder-The neurofeedback remedy. Biofeedback, 37(1), 24-31. http://dx.doi.org/10.5298/1081-5937-37.1.24

Othmer, S., \& Othmer, S. F. (2017). Toward a Frequency-Based Theory of Neurofeedback. In J. R. Evans \& R. A. Turner (Eds.), Rhythmic Stimulation Procedures in Neuromodulation (pp. 225-278), San Diego, CA: Academic Press. http://dx.doi.org/10.1016/B978-0-12-803726-3.00008-0

Othmer, S., Othmer, S. F., \& Kaiser, D. A. (1999). EEG biofeedback: An emerging model for its global efficacy. In J. R. Evans \& A. Abarbanel (Eds.), Introduction to quantitative EEG and neurofeedback (pp. 243-310). San Diego, CA: Academic Press.

Othmer, S., Othmer, S. F., Kaiser, D. A., \& Putman, J. (2013). Endogenous neuromodulation at infralow frequencies. Seminars in Pediatric Neurology and Psychiatry, 20(4), 246257. http://dx.doi.org/10.1016/j.spen.2013.10.006

Othmer, S., Othmer, S., \& Legarda, S. B. (2011). Clinical neurofeedback: Training brain behavior. Treatment Strategies - Pediatric Neurology and Psychiatry, 2(1), 67-73.

Othmer, S. F. (2017). Protocol Guide for Neurofeedback Clinicians (6th ed.). Los Angeles, CA: EEG Info. 
Pizzagalli, D. A., Oakes, T. R., \& Davidson, R. J. (2003). Coupling of theta activity and glucose metabolism in the human rostral anterior cingulate cortex: An EEG/PET study of normal and depressed subjects. Psychophysiology, 40(6), 939-949. http://dx.doi.org/10.1111/1469-8986.00112

Pollock, V. E., \& Schneider, L. S. (1990). Topographic quantitative EEG in elderly subjects with major depression. Psychophysiology, 27(4), 438-444. http://dx.doi.org/10.1111 /j.1469-8986.1990.tb02340.x

Porter, R. J., Gallagher, P., Thompson, J. M., \& Young, A. H. (2003). Neurocognitive impairment in drug-free patients with major depressive disorder. The British Journal of Psychiatry, 182(3), 214-220. http://dx.doi.org/10.1192/bjp.182.3.214

Raichle, M. E. (2011). The restless brain. Brain Connectivity, 1(1), 3-12. http://dx.doi.org/10.1089/brain.2011.0019

Roemer, R. A., Shagass, C., Dubin, W., Jaffe, R., \& Siegal, L. (1992). Quantitative EEG in elderly depressives. Brain Topography, 4(4), 285-290. http://dx.doi.org/10.1007 /BF01135566

Rosenfeld, J. P. (2000). An EEG biofeedback protocol for affective disorders. Clinical EEG and Neuroscience, 31(1), 712. http://dx.doi.org/10.1177/155005940003100106

Rosenfeld, J. P., Baehr, E., Baehr, R., Gotlib, I. H., \& Ranganath, C. (1996). Preliminary evidence that daily changes in frontal alpha asymmetry correlate with changes in affect in therapy sessions. International Journal of Psychophysiology, 23(1-2), 137-141. http://dx.doi.org/10.1016/0167-8760(96)00037-2

Schacter, D. L. (1977). EEG theta waves and psychological phenomena: A review and analysis. Biological Psychology, 5(1), 47-82. http://dx.doi.org/10.1016/0301-0511(77)90028-X

Shankman, S. A., \& Klein, D. N. (2003). The relation between depression and anxiety: An evaluation of the tripartite, approach-withdrawal and valence-arousal models. Clinical Psychology Review, 23(4), 605-637. http://dx.doi.org/10.1016 IS0272-7358(03)00038-2

Sterman, M. B., \& Friar, L. (1972). Suppression of seizures in an epileptic following sensorimotor EEG feedback training. Electroencephalography and Clinical Neurophysiology, 33(1), 89-95. http://dx.doi.org/10.1016/0013-4694(72)90028-4
Strijkstra, A. M., Beersma, D. G., Drayer, B., Halbesma, N., \& Daan, S. (2003). Subjective sleepiness correlates negatively with global alpha $(8-12 \mathrm{~Hz})$ and positively with central frontal theta $(4-8 \mathrm{~Hz})$ frequencies in the human resting awake electroencephalogram. Neuroscience Letters, 340(1), 17-20.

Suffin, S. C., \& Emory, W. H. (1995). Neurometric subgroups in attentional and affective disorders and their association with pharmacotherapeutic outcome. Clinical EEG and Neuroscience, 26(2), 76-83. http://dx.doi.org/10.1177 $/ 155005949502600204$

Tomarken, A. J., \& Keener, A. D. (1998). Frontal brain asymmetry and depression: A self-regulatory perspective. Cognition and Emotion, 12(3), 387-420. http://dx.doi.org/10.1080 1026999398379655

Vanhatalo, S., Palva, J. M., Holmes, M. D., Miller, J. W., Voipio, J., \& Kaila, K. (2004). Infraslow oscillations modulate excitability and interictal epileptic activity in the human cortex during sleep. Proceedings of the National Academy of Sciences of the United States of America, 101(14), 50535057. http://dx.doi.org/10.1073/pnas.0305375101

Van Putten, M. J. A. M., Tjepkema-Cloostermans, M. C., \& Hofmeijer, J. (2015). Infraslow EEG activity modulates cortical excitability in postanoxic encephalopathy. Journal of Neurophysiology, 113(9), 3256-3267. http://dx.doi.org /10.1152/jn.00714.2014

Volf, N. V., \& Passynkova, N. R. (2002). EEG mapping in seasonal affective disorder. Journal of Affective Disorders, 72(1), 61-69. http://dx.doi.org/10.1016/S01650327(01)00425-6

Yamada, M., Kimura, M., Mori, T., \& Endo, S. (1995). EEG power and coherence in presenile and senile depression. Characteristic findings related to differences between anxiety type and retardation type. Nihon Ika Daigaku Zasshi, 62(2), 176-185. http://dx.doi.org/10.1272/jnms1923.62.176

Received: March 2, 2018

Accepted: March 27, 2018

Published: March 31, 2018 